\title{
Ergänzung zum Abstract Hauch T et al. Ultraschall in Med 2012; 33: S109
}

Im Abstract-Heft für das Dreiländertreffen 2012 in Davos erfolgte beim folgenden Abstract leider eine unvollständige Nennung der Autoren. Diese seien hiermit nachgetragen:

Wie hoch ist der Behalteeffekt nach Frontalpräsentationen von Ultraschallkursen? - „Death by Powerpoint"

$\nabla$

Hauch $\mathrm{T}^{1,2}$, Stenger $\mathrm{D}^{3}$, Seibel $\mathrm{A}^{4}$, Armbruster $\mathrm{W}^{5}$, Heringer $\mathrm{F}^{2}$, Walcher $\mathrm{F}^{2}$, Schellhaas $\mathrm{S}^{2,6}$, Breitkreutz $\mathrm{R}^{2,7}$

${ }^{1}$ Abteilung für Innere Medizin, Städtisches Klinikum, Neunkirchen/Saar, Deutschland

${ }^{2}$ Frankfurter interdisziplinäres Institut für Notfallmedizin und Simulationstraining (FINeST)

${ }^{3}$ Klinik für Unfall-, Hand- und Wiederherstellungschirurgie, Universitätsklinikum des Saarlandes, Homburg/Saar, Deutschland

${ }^{4}$ Abteilung für Anästhesie, Intensivmedizin und Notfallmedizin, Diakonie Klinikum, Siegen, Deutschland

${ }^{5}$ Klinik für Anästhesiologie, Intensivmedizin, Schmerztherapie, Evangelisches Krankenhaus, Unna, Deutschland ${ }^{6}$ Abteilung für Kardiologie, Alice-Hospital, Darmstadt, Deutschland

${ }^{7}$ Zentrale Notaufnahme, Klinikum der Stadt Frankfurt am Main (Höchst), Deutschland 